\title{
Self-Attentive Model for Headline Generation
}

\author{
Daniil Gavrilov, Pavel Kalaidin, and Valentin Malykh \\ VK, \\ 191023, Nevsky ave., 28, Saint-Petersburg, Russia \\ \{firstname.lastname\}@vk.com
}

\begin{abstract}
Headline generation is a special type of text summarization task. While the amount of available training data for this task is almost unlimited, it still remains challenging, as learning to generate headlines for news articles implies that the model has strong reasoning about natural language. To overcome this issue, we applied recent Universal Transformer architecture paired with byte-pair encoding technique and achieved new state-of-the-art results on the New York Times Annotated corpus with ROUGE-L F1-score 24.84 and ROUGE-2 F1-score 13.48. We also present the new RIA corpus and reach ROUGE-L F1-score 36.81 and ROUGE-2 F1-score 22.15 on it.
\end{abstract}

Keywords: universal transformer $\cdot$ headline generation $\cdot$ BPE · summarization.

\section{Introduction}

Headline writing style has broader applications than those used purely within the journalism community. So-called naming is one of the arts of journalism. Just as natural language processing techniques help people with tasks such as incoming message classification (see [5] or [6]), the naming problem could also be solved using modern machine learning and, in particular, deep learning techniques. In the field of machine learning, the naming problem is formulated as headline generation, i.e. given the text it is needed to generate a title.

Headline generation can also be seen as a special type of text summarization. The aim of summarization is to produce a shorter version of the text that captures the main idea of the source version. We focus on abstractive summarization when the summary is generated on the fly, conditioned on the source sentence, possibly containing novel words not used in the original text.

The downside of traditional summarization is that finding a source of summaries for a large number of texts is rather costly. The advantage of headline generation over the traditional approach is that we have an endless supply of news articles since they are available in every major language and almost always have a title.

This task could be considered language-independent due to the absence of the necessity of native speakers for markup and/or model development.

While the task of learning to generate article headlines may seem to be easier than generating full summaries, it still requires that the learning algorithm be 
able to catch structure dependencies in natural language and therefore could be an interesting benchmark for testing various approaches.

In this paper, we present a new approach to headline generation based on Universal Transformer architecture which explicitly learns non-local representations of the text and seems to be necessary to train summarization model. We also present the test results of our model on the New York Times Annotated corpus and the RIA corpus.

\section{Related Work}

Rush et al. [1] were the first to apply an attention mechanism to abstractive text summarization.

In the recent work of Hayashi [4], an encoder-decoder approach was presented, where the first sentence was reformulated to a headline. Our Encoder-Decoder baseline (see section 6.1) follows their setup.

The related approach was presented in [10, where the approach of the first sentence was expanded with a so-called topic sentence. The topic sentence is chosen to be the first sentence containing the most important information from a news article (so called $5 \mathrm{~W} 1 \mathrm{H}$ information, where $5 \mathrm{~W} 1 \mathrm{H}$ stands for who, what, where, when, why, how). Our Encoder-Decoder baseline could be considered to implement their approach in OF (trained On First sentence) setup.

Tan et al. in [15] present an encoder-decoder approach based on a pregenerated summary of the article. The summary is generated using a statistical summarization approach. The authors mention that the first sentence approach is not enough for New York Times corpora, but they only use a summary for their approach instead of the whole text, thus relying on external tools of summarization.

\section{Background}

Consider that we have dataset $D=\left\{\left(\text { title }_{i}, \text { fulltext }_{i}\right)\right\}_{i}^{N}$ of news articles and their titles. An approach for learning summarization is to define a conditional probability $P\left(y_{t} \mid\left\{y_{1}, \ldots, y_{t-1}\right\}, X, \theta\right)$ of some token $y_{t} \in V$ at time step $t \in \mathbb{N}$, with respect to article text $X=\left\{x_{1}, \ldots, x_{N}\right\}\left(x_{i} \in V\right.$ too) and previous tokens of the title $\left\{y_{1}, \ldots, y_{t-1}\right\}$, parameterized by a neural network with parameters $\theta$.

Then model parameters are found as $\theta_{M L E}=\operatorname{argmax}_{\theta} \prod_{i}^{N} P\left(Y_{i} \mid X_{i}, \theta\right)$

We can then apply two methods for finding the most probable sentence under trained model: greedy, decoding token-by-token by finding the most probable token at each time step, and beam-search, where we find the top-k most probable tokens at each step. The latter method yields better results though it is more computationally expensive.

Sutskever et al. [14] proposed a model that defines $P\left(y_{t} \mid\left\{y_{1}, \ldots, y_{t-1}\right\}, X, \theta\right)$ by propagating initial sequence $X$ through a Recurrent Neural Network (RNN). Then last hidden state of RNN is used as context vector $c$ and is then passed to the second RNN with $y_{1}, \ldots, y_{t-1}$ to obtain distribution over $y_{t}$. 
RNNs have a commonly known flaw. They rapidly forget earlier timesteps, e.g. see [2]. To mitigate this issue, attention [1] was introduced to the EncoderDecoder architecture. The attention mechanism makes a model able to obtain a new context vector at every decoding iteration from different parts of an encoded sequence. It helps capture all the relevant information from the input sequence, removing the bottleneck of the fixed size hidden vector of the decoder's RNN.

\section{Our Approach}

\subsection{Universal Transformer}

While RNNs could be easily used to define the Encoder-Decoder model, learning the recurrent model is very expensive from a computation perspective. The other drawback is that they use only local information while omitting a sequence of hidden states $H=\left\{h_{1}, \ldots, h_{N}\right\}$. I.e. any two vectors from hidden state $h_{i}$ and $h_{j}$ are connected with $j-i$ RNN computations that makes it hard to catch all the dependencies in them due to limited capacity. To train a rich model that would learn complex text structure, we have to define a model that relies on non-local dependencies in the data.

In this work, we adopt the Universal Transformer model architecture [3], which is a modified version of Transformer [16. This approach has several benefits over RNNs. First of all, it could be trained in parallel. Furthermore, all input vectors are connected to every other via the attention mechanism. It implies that Transformer architecture learns non-local dependencies between tokens regardless of the distance between them, and thus it is able to learn a more complex representation of the text in the article, which proves to be necessary to effectively solve the task of summarization. Also, unlike [4|15], our model is trained end-to-end using the text and title of each news article.

\subsection{Byte Pair Encoding}

We also adopt byte-pair encoding (BPE), introduced by Sennrich for the machine translation task in [13. BPE is a data compression technique where often encountered pairs of bytes are replaced by additional extra-alphabet symbols. In the case of texts, like in the machine translation field, the most frequent words are kept in the vocabulary, while less frequent words are replaced by a sequence of (typically two) tokens. E.g., for morphologically rich languages, the word endings could be detached since each word form is definitely less frequent than its stem. BPE encoding allows us to represent all words, including the ones unseen during training, with a fixed vocabulary.

\section{Experiments}

In our experiments, we consider two corpora: one in Russian and another in English. It is important to mention that we have not done any additional preprocessing other than lower casing, unlike other approaches [410]. We apply 
BPE encoding, which allows us to avoid usage of the $\langle U N K\rangle$ token for outof-vocabulary words. For our experiments, we withheld 20,000 random articles to form the test set. We have repeated our experiments 5 times with different random seeds and report mean values.

English Dataset We use the New York Times Annotated Corpus (NYT) as presented by the Linguistic Data Consortium in [12. This dataset contains 1.8 million news articles from the New York Times news agency, written between the years 1987 and 2006. For our experiments, we filtered out news articles containing titles shorter than 3 words or longer than 15 words. We also filtered articles with a body text shorter than 20 words or longer than 2000 words. In addition, we skipped obituaries in the dataset. After filtering, we had 1444919 news available to us with a mean title length of 7.9 words and mean text length of 707.6 words.

Russian Dataset Russian news agency "Rossiya Segodnya" provided us with a dataset (RIA) for research purposes 1 . It contains news documents from January, 2010 to December, 2014. In total, there are 1003869 news articles in the provided corpus with a mean title length 9.5 words and mean text length of 315.6 words.

\section{Experiments}

\subsection{Baseline models}

First Sentence This model takes the first sentence of an article and uses it as its hypothesis for an article headline. This is a strong baseline for generating headlines from news articles.

Encoder-Decoder Following [10], we use the encoder-decoder architecture on the first sentence of an article. The model itself is already described at recent works section as Seq-To-Seq with RNNs of Sutskever et al. [14. For this approach, we use the same preprocessing as we did for our model, including byte pair encoding.

\subsection{Training}

For both datasets, NYT and RIA, we used the same set of hyper-parameters for the models, namely 4 layers in the encoder and decoder with 8 heads of attention. In addition, we added a Dropout of $p=0.3$ before applying Layer Normalization [8].

The models were trained with the Adam optimizer using a scaled learning rate, as proposed by the authors of the original Transformer with the number

\footnotetext{
${ }^{1}$ The dataset is available at https://vk.cc/8W015P
} 
of warmout steps equal to 4000 in both cases and $\beta=(0.9,0.98)$. Both models were trained until convergence.

We trained the BPE tokenizator separately on the datasets. NYT data was tokenized with a vocabulary size of active tokens equal to 40000, while RIA data was tokenized using 50000 token vocabulary. In addition, we have limited length of the documents with 3000 BPE tokens and 2000 BPE tokens for RIA and NYT datasets respectively. Any exceeding tokens were omitted. word2vec [9] embeddings were trained on each dataset with the size of each embedding equal to 512. For headline generation, we adopted beam-search size of 10 .

\section{Results}

\begin{tabular}{|l|cccccc|}
\hline Model & R-1-f & R-1-r & R-2-f & R-2-r & R-L-f & R-L-r \\
\hline \multicolumn{7}{|c|}{ New York Times } \\
\hline First Sentence & 11.64 & $\mathbf{3 4 . 6 7}$ & 2.28 & 7.43 & 7.19 & $\mathbf{3 1 . 3 9}$ \\
Encoder-Decoder & 23.02 & 21.90 & 11.84 & 11.44 & 21.23 & 21.31 \\
summ-hieratt [15] & - & 29.60 & - & 8.17 & - & 26.05 \\
Universal Transformer w/ smoothing (ours) & 25.60 & 23.90 & 12.92 & 12.42 & 23.66 & 25.27 \\
Universal Transformer (ours) & $\mathbf{2 6 . 8 6}$ & 25.33 & $\mathbf{1 3 . 4 8}$ & $\mathbf{1 3 . 0 1}$ & $\mathbf{2 4 . 8 4}$ & 24.38 \\
\hline \multicolumn{7}{|c|}{ Rossiya Segodnya } \\
\hline First Sentence & 24.08 & $\mathbf{4 5 . 5 8}$ & 10.57 & 21.30 & 16.70 & $\mathbf{4 1 . 6 7}$ \\
Encoder-Decoder & 39.10 & 38.31 & 22.13 & $\mathbf{2 1 . 7 5}$ & 36.34 & 36.34 \\
Universal Transformer w/ smoothing (ours) & 39.31 & 37.10 & 21.82 & 20.66 & 36.32 & 35.37 \\
Universal Transformer (ours) & $\mathbf{3 9 . 7 5}$ & 37.62 & $\mathbf{2 2 . 1 5}$ & 21.04 & $\mathbf{3 6 . 8 1}$ & 35.91 \\
\hline
\end{tabular}

Table 1. ROUGE-1,2,L $F_{1}$ and recall scores, on NYT corpus and RIA corpus.

In Tab. 11 we present results based on two corpora: the New York Times Annotated (NYT) corpus for English, and the Rossiya Segodnya (RIA) corpus for Russian. For the NYT corpus, we reached a new state of the art on ROUGE1, ROUGE-2 and ROUGE-L $F_{1}$ scores. For the RIA corpus, since it has no previous art, we present results for the baselines and our model 2 For our model we also experimented with label smoothing following [7].

In our experiments, we noticed that some of the generated headlines are scored low by ROUGE metrics despite seeming reasonable, e.g. top sample in Tab. 3. This lead us to a new series of experiments. We conducted human evaluation of obtained results for both NYT and RIA corpora. The results are presented

\footnotetext{
${ }^{2}$ We are providing results from Tan et al. [15], which were achieved using the NYT corpus. Unfortunately, the authors have not published all of their filtering criteria and seed for random sampling for this corpus, so we could not follow their setup completely. Therefore, these results are presented here for reference.
} 


\begin{tabular}{|l|ccc|}
\hline User Preference & Human & Tie & Machine \\
\hline Dataset & 57.4 & 27.4 & 15.2 \\
Rew York Times Annotated & 54.4 & 30.6 & 15.0 \\
\hline
\end{tabular}

Table 2. Human evaluation results for NYT and RIA datasets.

in Tab. 2. 5 annotators marked up 100 randomly sampled articles from a train set of each corpora. Each number shows the percentage of annotator preference over three possible options: original headline (Human), generated headline (Machine), no preference (Tie).

For the both corpora, we could see that our model is not reaching human parity yet, having $42.6 \%$ and $45.6 \%$ of (Machine + Tie) user preference for NYT and RIA datasets respectively, but this result is already close to human parity and leaves room for improvement.

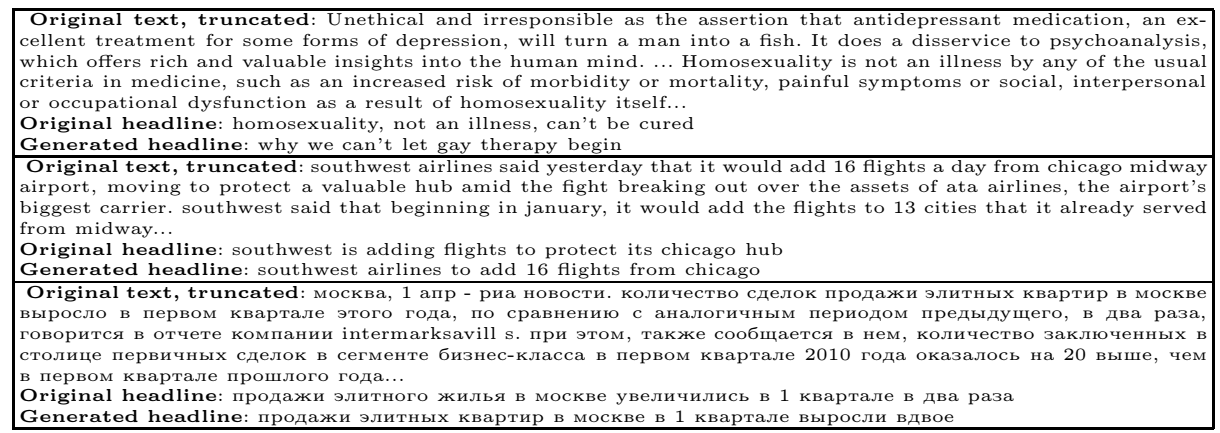

Table 3. Samples of headlines generated by our model.

\section{Conclusion}

In this paper, we explore the application of Universal Transformer architecture to the task of abstractive headline generation and outperform the abstractive stateof-the-art result on the New York Times Annotated corpus. We also present a newly released Rossiya Segodnya corpus and results achieved by our model applied to it.

Acknowledgments. Authors are thankful to Alexey Samarin for useful discussions, David Prince for proofreading, Madina Kabirova for proofreading and human evaluation organization, Anastasia Semenyuk and Maria Zaharova for help obtaining the New York Times Annotated corpus, and Alexey Filippovskii for providing the Rossiya Segodnya corpus. 


\section{References}

1. Bahdanau, D., Cho, K., Bengio, Y.: Neural machine translation by jointly learning to align and translate. arXiv preprint arXiv:1409.0473 (2014)

2. Bengio, Y., Simard, P., Frasconi, P.: Learning long-term dependencies with gradient descent is difficult. IEEE Transactions on Neural Networks 5(2), 157-166 (Mar 1994). https://doi.org/10.1109/72.279181

3. Dehghani, M., Gouws, S., Vinyals, O., Uszkoreit, J., Kaiser, Ł.: Universal transformers. arXiv preprint arXiv:1807.03819 (2018)

4. Hayashi, Y., Yanagimoto, H.: Headline generation with recurrent neural network. In: New Trends in E-service and Smart Computing, pp. 81-96. Springer (2018)

5. Howard, J., Ruder, S.: Fine-tuned language models for text classification. arXiv preprint arXiv:1801.06146 (2018)

6. Joulin, A., Grave, E., Bojanowski, P., Mikolov, T.: Bag of tricks for efficient text classification. In: Proceedings of the 15th Conference of the European Chapter of the Association for Computational Linguistics: Volume 2, Short Papers. vol. 2, pp. 427-431 (2017)

7. Kim, B., Kim, H., Kim, G.: Abstractive Summarization of Reddit Posts with Multilevel Memory Networks. In: arXiv (2018)

8. Lei Ba, J., Ryan Kiros, J., Hinton, G.: Layer normalization (2016)

9. Mikolov, T., Sutskever, I., Chen, K., Corrado, G., Dean, J.: Distributed representations of words and phrases and their compositionality. In Proceedings of NIPS (2013)

10. Putra, J.W.G., Kobayashi, H., Shimizu, N.: Experiment on using topic sentence for neural news headline generation (2018)

11. Rush, A.M., Chopra, S., Weston, J.: A neural attention model for abstractive sentence summarization. In: Empirical Methods in Natural Language Processing. pp. 379-389 (2015)

12. Sandhaus, E.: The new york times annotated corpus ldc2008t19. dvd. Linguistic Data Consortium, Philadelphia (2008)

13. Sennrich, R., Haddow, B., Birch, A.: Neural machine translation of rare words with subword units. https://arxiv.org/abs/1508.07909 (2015)

14. Sutskever, I., Vinyals, O., Le, Q.V.: Sequence to sequence learning with neural networks. https://arxiv.org/abs/1409.3215 (2014)

15. Tan, J., Wan, X., Xiao, J.: From neural sentence summarization to headline generation: a coarse-to-fine approach. In: Proceedings of the 26th International Joint Conference on Artificial Intelligence. pp. 4109-4115. AAAI Press (2017)

16. Vaswani, A., Shazeer, N., Parmar, N., Uszkoreit, J., Jones, L., Gomez, A.N., Kaiser, Ł., Polosukhin, I.: Attention is all you need. In: Advances in Neural Information Processing Systems. pp. 5998-6008 (2017) 